\title{
Operators of equivalent sorting power and related Wilf-equivalences
}

\author{
Michael Albert ${ }^{1}$ and Mathilde Bouvel $\|^{2}$ \\ ${ }^{1}$ Department of Computer Science, University of Otago, Dunedin, New Zealand \\ ${ }^{2}$ LaBRI, CNRS and Univ. Bordeaux, Talence, France.
}

\begin{abstract}
We study sorting operators A on permutations that are obtained composing Knuth's stack sorting operator $\mathbf{S}$ and the reverse operator $\mathbf{R}$, as many times as desired. For any such operator $\mathbf{A}$, we provide a bijection between the set of permutations sorted by $\mathbf{S} \circ \mathbf{A}$ and the set of those sorted by $\mathbf{S} \circ \mathbf{R} \circ \mathbf{A}$, proving that these sets are enumerated by the same sequence, but also that many classical permutation statistics are equidistributed across these two sets. The description of this family of bijections is based on an apparently novel bijection between the set of permutations avoiding the pattern 231 and the set of those avoiding 132 which preserves many permutation statistics. We also present other properties of this bijection, in particular for finding families of Wilf-equivalent permutation classes.

Résumé. On étudie les opérateurs $\mathbf{A}$ de tri de permutations obtenus en composant l'opérateur $\mathbf{S}$ de tri par une pile de Knuth et l'opérateur $\mathbf{R}$ de miroir, un certain nombre de fois. Pour tout opérateur $\mathbf{A}$ de cette forme, on donne une bijection entre l'ensemble des permutations triées par $\mathbf{S} \circ \mathbf{A}$ et l'ensemble de celles triées par $\mathbf{S} \circ \mathbf{R} \circ \mathbf{A}$, démontrant ainsi que ces ensembles ont la même séquence d'énumération, mais aussi que de nombreuses statistiques classiques sur les permutations ont la même distribution sur ces deux ensembles. La description de cette famille de bijections repose sur une bijection apparemment nouvelle entre l'ensemble des permutations qui évitent le motif 231 et l'ensemble de celles qui évitent 132, qui préserve de nombreuses statistiques. On présente aussi d'autres propriétés de cette bijection, en particulier pour trouver des familles de classes de permutations équivalentes au sens de Wilf.
\end{abstract}

Keywords: permutation, stack, sorting, enumeration, Wilf-equivalence

\section{Introduction}

Partial sorting algorithms were one of the early motivations for the study of permutation patterns. For instance, Knuth (1975) considered the problem of sorting a permutation of length $n$, i.e. of the set $[n]=$ $\{1,2, \ldots, n\}$, using only a stack. If such a permutation, $\pi$, is written in one line notation as $\alpha n \beta$, then $\pi$ is sortable if and only if: each of $\alpha$ and $\beta$ is sortable (thought of as permutations of the values they contain); and each value in $\alpha$ is less than any value in $\beta$ (or simply $\alpha<\beta$ ). The first condition is clearly necessary - the second condition is also necessary as, when $n$ is the first element remaining to be added to the stack, the entire stack must be emptied to have any hope of success, otherwise $n$ will precede some

\footnotetext{
${ }^{\dagger}$ Partially supported by ANR projects MAGNUM (2010-BLAN-0204) and PSYCO (ANR-11-JS02-001). 
other element in the output, and the output will not be sorted. In the same fashion, the stack must at all times obey the Hanoi condition that it never has a greater element lying on top of a lesser one. That the conditions are sufficient is also clear - the requisite operations are: sort and output $\alpha$; add $n$ to the stack; sort and output $\beta$; remove $n$ from the stack. Figure 1 shows an example of performing stack sorting on a permutation. This simple behavior prompted many other investigations of stack sorting and its variations and extensions beginning with works by Pratt(1973) and Tarjan (1972).
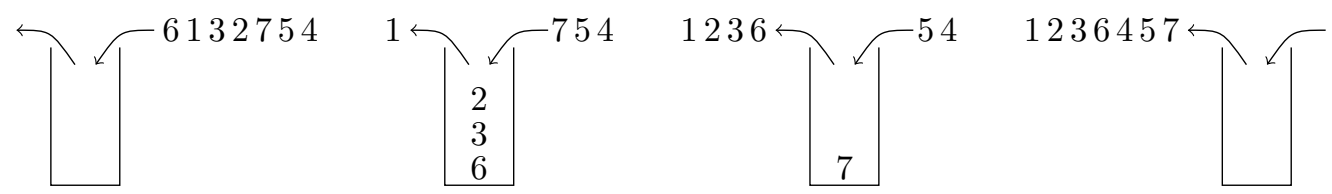

Fig. 1: Some steps of the stack sorting procedure applied to $\pi=6132754$. Thus, $\mathbf{S}(\pi)=1236457$.

Stack sorting can be considered as an operator or procedure, $\mathbf{S}$, applied to permutations. It is defined recursively as: $\mathbf{S}(\alpha n \beta)=\mathbf{S}(\alpha) \mathbf{S}(\beta) n$. With this definition $\mathbf{S}(\pi)$ is the result of attempting to sort $\pi$ using a stack, maintaining the condition that the items in the stack must always be ordered from least to greatest when read from top to bottom. We adopt the viewpoint throughout that any sequence of distinct values can be interpreted as a permutation and " $n$ " always denotes the maximum element of such a sequence. West (1993) described the permutations that can be sorted using $\mathbf{S} \circ \mathbf{S}$, and Zeilberger (1992) subsequently confirmed a conjecture of West's on their enumeration.

Bousquet-Mélou (2000) also considered the operator $\mathbf{S}$ and characterized, given $\pi$, the set $\mathbf{S}^{-1}(\pi)$. We shall be extending her results, and will discuss them in more detail later. Central to her analysis is the observation that the operator $\mathbf{S}$ can be described in the following terms: given a permutation $\pi$ form the unique decreasing binary tree $T_{\text {in }}(\pi)$ whose in-order reading is $\pi$, then $\mathbf{S}(\pi)$ is the post-order reading of this tree.

A second operator on permutations is the reversal operator, that reads permutations from right to left - it can also be modeled by using a stack where we are obliged to input the entire permutation to the stack before performing any output. The reversal operator, $\mathbf{R}$, is one of eight natural symmetries on the collection of permutations. Bouvel and Guibert (2012) considered the enumeration of permutations sorted by $\mathbf{S} \circ \mathbf{R} \circ \mathbf{S}$ as well as the sets defined similarly with other symmetries in place of $\mathbf{R}$. In experimental investigations aimed at providing extensions to their results they noticed an interesting phenomenon that can be expressed as:

Conjecture $\mathbf{1}$ For any composition, $\mathbf{A}$, of the operators $\mathbf{S}$ and $\mathbf{R}$ the number of permutations sorted by $\mathbf{S} \circ \mathbf{A}$ and by $\mathbf{S} \circ \mathbf{R} \circ \mathbf{A}$ is the same. Moreover, many permutation statistics are equidistributed across these two sets.

It is the primary purpose of this article to prove that this is indeed the case. To do so, we make use of another classical description of stack sortable permutations. It is simply derived from their description by Knuth (1975) that we reported at the beginning of this section. Stack sortable permutations are those that may not contain subwords (not necessarily consecutive) of the form $b c a$ where $a<b<c$. Such permutations are said to avoid the pattern 231, and the collection of all such is denoted $\operatorname{Av}(231)$. More generally and more formally, a permutation $\pi=\pi(1) \pi(2) \cdots \pi(k)$ is a pattern of a permutation 
$\sigma=\sigma(1) \sigma(2) \cdots \sigma(n)$ when there exist $1 \leq i_{1}<i_{2}<\cdots<i_{k} \leq n$ such that $\pi$ is order isomorphic to $\sigma\left(i_{1}\right) \sigma\left(i_{2}\right) \cdots \sigma\left(i_{k}\right)$. If $\pi$ is not a pattern of $\sigma$ then we say that $\sigma$ avoids $\pi$. We denote by $\operatorname{Av}\left(\pi, \pi^{\prime}, \cdots, \pi^{\prime \prime}\right)$ the set of all permutations that avoid simultaneously the patterns $\pi, \pi^{\prime}, \cdots, \pi^{\prime \prime}$. Such a collection of permutations defined by the avoidance of a given set of permutations is also called a permutation class.

With the characterization of stack sortable permutations as $\operatorname{Av}(231)$, proving Conjecture 1 is equivalent to showing that there is a bijection between the elements of $\operatorname{Av}(231)$ belonging to the image of $\mathbf{A}$, and the elements of $\operatorname{Av}(231)$ belonging to the image of $\mathbf{R} \circ \mathbf{A}$, with the additional condition that the bijection preserves the number of preimages under $\mathbf{A}$ (resp. $\mathbf{R} \circ \mathbf{A}$ ). Equivalently, we can replace this latter set by the elements of $\operatorname{Av}(132)$ belonging to the image of $\mathbf{A}$, since the self-inverse operator $\mathbf{R}$ immediately provides a bijection between $\mathrm{Av}(231)$ and $\mathrm{Av}(132)$.

In establishing this result we demonstrate an apparently novel bijection between $\operatorname{Av}(231)$ and $\operatorname{Av}(132)$ which preserves many permutation statistics. We also present some other properties of this bijection.

\section{Preimages of permutations in the image of $\mathrm{S}$}

As noted earlier, the description of the elements of $\mathbf{S}^{-1}(\pi)$ for $\pi$ in the image of $\mathbf{S}$ was carried out by Bousquet-Mélou (2000). This description is central to our work, so we review it here.

There exists for any permutation $\sigma$ a unique decreasing binary tree, $\mathrm{T}_{\mathrm{in}}(\sigma)$ whose in-order reading is $\sigma$. As usual, $\mathrm{T}_{\mathrm{in}}(\sigma)$ is recursively defined: if $\sigma=\alpha n \beta$ then the root of $\mathrm{T}_{\mathrm{in}}(\sigma)$ is $n$ and its left (resp. right) subtrees are $\mathrm{T}_{\mathrm{in}}(\alpha)$ (resp. $\mathrm{T}_{\mathrm{in}}(\beta)$ ). The recursive description of $\mathbf{S}$ given above $(\mathbf{S}(\alpha n \beta)=\mathbf{S}(\alpha) \mathbf{S}(\beta) n)$ then shows that $\mathbf{S}$ converts in-order reading of decreasing binary trees to post-order reading. Therefore, describing $\mathbf{S}^{-1}(\pi)$ is equivalent to describing the decreasing binary trees, $T$, with post-order reading $\pi$. For convenience we denote the post-order reading of a tree $T$ by $\operatorname{Post}(T)$.

Definition 2 A decreasing binary tree is canonical if it has the following property: any node, z, that has a left child, $x$, also has a right child, and the leftmost value $y$ in the subtree of the right child of $z$ is less than $x$.

From (Bousquet-Mélou, 2000, Proposition 2.6), we know that for $\pi$ in the image of $\mathbf{S}$ there is a unique canonical tree $T_{\pi}$ with $\operatorname{Post}\left(T_{\pi}\right)=\pi$. In fact, the permutation $\sigma$ obtained from the in-order reading of $T_{\pi}$ is the element of $\mathbf{S}^{-1}(\pi)$ having the greatest number of inversions. Moreover, any decreasing binary tree whose post-order reading is $\pi$ (and only such trees) can be obtained from $T_{\pi}$ by a sequence of operations of the following type: take a node $z$ with no left child, and one of its descendants $y$ on the leftmost branch of its right subtree; remove the subtree rooted at $y$ and make it the left subtree of $z$. It follows that $\left|\mathbf{S}^{-1}(\pi)\right|$ depends only on the structure of the tree $T_{\pi}$ and not on its labeling.

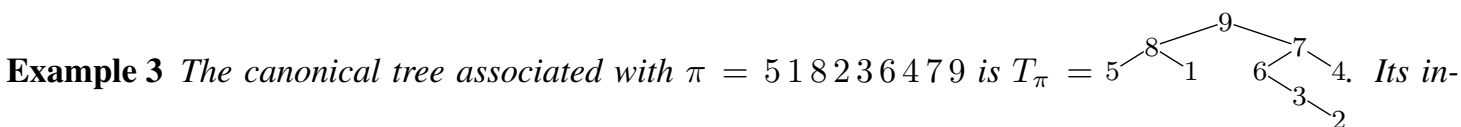
order reading, $\sigma=581963274$ gives the permutation with the largest number of inversions subject to $\mathbf{S}(\sigma)=\pi$. The four other decreasing binary trees with the same post-order reading are shown in Figure 2. Thus $\left|\mathbf{S}^{-1}(\pi)\right|=5$. If the labels 8 and 7 , and 5 and 4 , were exchanged in the original tree, corresponding to $\pi^{\prime}=417236589$ then, because the tree is still canonical, the method for constructing permutations in $\mathbf{S}^{-1}\left(\pi^{\prime}\right)$ is still the same, and in particular $\left|\mathbf{S}^{-1}\left(\pi^{\prime}\right)\right|=\left|\mathbf{S}^{-1}(\pi)\right|$. 

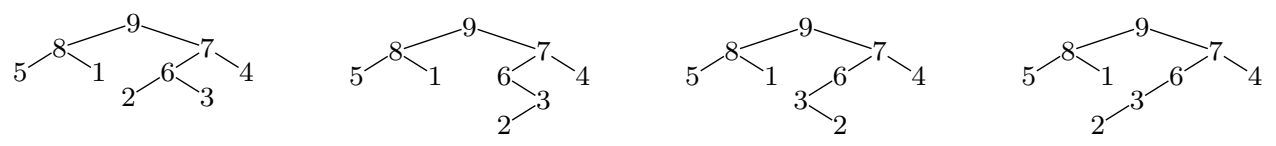

Fig. 2: The four non canonical decreasing trees whose post-order reading is $\pi=518236479$.

\section{A recursive bijection between $\operatorname{Av}(231)$ and $\operatorname{Av}(132)$}

In this section we introduce a bijection, $P$, between permutations in $\operatorname{Av}(231)$ and those in $\operatorname{Av}(132)$. It is very easy to describe $P$ recursively using the sum, $\oplus$, and skew sum, $\ominus$, operations on permutations. These operations are easily understood on the diagrams corresponding to permutations. The diagram of any permutation $\sigma$ of length $n$ is the set of $n$ points in the plane at coordinates $(i, \sigma(i))$. If $\alpha$ is a permutation of $[a]$ and $\beta$ of $[b]$ we define:

$$
\begin{aligned}
& \alpha \oplus \beta=\alpha(\beta+a) \text { whose diagram is } \alpha \\
& \alpha \ominus \beta=(\alpha+b) \beta \text { whose diagram is } \frac{\alpha}{\alpha} .
\end{aligned}
$$

Here for example $\beta+a$ is just the sequence obtained by adding $a$ to every element of the sequence $\beta$ and $\alpha$ represents the diagram of permutation $\alpha$.

Example 4 Let $\alpha=231$ and $\beta=3142$. Then $\alpha \oplus \beta=2316475$, while $\alpha \ominus \beta=6753142$.

Any permutation $\sigma$ that can be written as a sum $\alpha \oplus \beta$ (resp. skew sum $\alpha \ominus \beta$ ) is said $\oplus$-decomposable (resp. $\ominus$-decomposable). Otherwise, we say that $\sigma$ is $\oplus$-indecomposable (resp. $\ominus$-indecomposable).

Any $\pi \in \operatorname{Av}(231)$ is either the empty permutation $\varepsilon$ or has a unique decomposition in the form $\alpha \oplus$ $(1 \ominus \beta)$ where $\alpha, \beta \in \operatorname{Av}(231)$ (and are possibly empty), and conversely any permutation of this latter form lies in $\operatorname{Av}(231)$. This is simply because the elements preceding the maximum in a 231-avoiding permutation must all be less than those following the maximum, and the prefix before and suffix after the maximum must also avoid 231. Conversely, if a permutation has this structure it cannot involve 231. This decomposition makes it easy to define the bijection $P$ recursively: $P(\varepsilon)=\varepsilon$ and

$$
\text { if } \pi=\alpha \oplus(1 \ominus \beta) \text { then } P(\pi)=(P(\alpha) \oplus 1) \ominus P(\beta) \text {. }
$$

Alternatively, with diagrams:

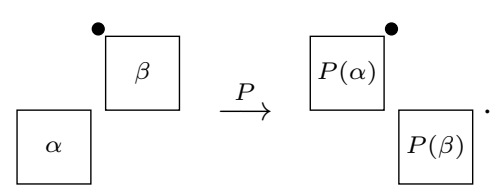

As the 132-avoiding permutations have a generic decomposition of the form shown on the right above, and since $P(1)=1$ maps the unique 231-avoiding permutation of length 1 to the unique 132-avoiding permutation of length 1 , induction immediately implies that $P: \operatorname{Av}(231) \rightarrow \operatorname{Av}(132)$ is a bijection. Notice that the restriction of $P$ to the set $\operatorname{Av}(231,132)$ is the identity map.

Example 5 For $\pi=153249867 \in \operatorname{Av}(231)$, we have $P(\pi)=785469312$. 
We recall a definition from the introduction:

Definition 6 For any permutation $\pi, \mathrm{T}_{\mathrm{in}}(\pi)$ is the decreasing binary tree whose in-order reading is $\pi$.

It follows immediately by induction from the recursive description of $P$ that:

Observation 7 Both $\mathrm{T}_{\mathrm{in}}(\pi)$ and $\mathrm{T}_{\mathrm{in}}(P(\pi))$ have the same underlying unlabeled tree, or briefly " $P$ preserves the shape of in-order trees". An example is provided in Figure 3
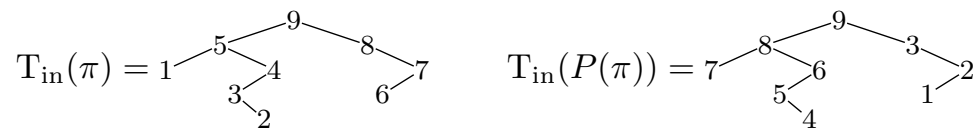

Fig. 3: $\mathrm{T}_{\mathrm{in}}(\pi)$ and $\mathrm{T}_{\mathrm{in}}(P(\pi))$ for the permutation $\pi=153249867$ of Example 5

It is for this reason that $P$ preserves many permutation statistics. Recall that, for $\pi$ a permutation of length $n$, a left-to-right (resp. right-to-left) maximum of $\pi$ is an element $\pi(i)$ such that for all $j<i$ (resp. $j>i$ ), $\pi(j)<\pi(i)$, and that the up-down word of $\pi$ is $w_{\pi} \in\{u, d\}^{n-1}$ with $w_{\pi}(i)=u$ (resp. $d$ ) if $\pi(i)<\pi(i+1)$ (resp. $\pi(i)>\pi(i+1)$ ).

Observation 8 Preserves the following statistics: the number and positions of the right-to-left maxima, the number and positions of the left-to-right maxima and the up-down word.

Proof: All of these follow from Observation 7 , since the value of each statistic mentioned for a permutation $\pi$ is determined by the shape of $\mathrm{T}_{\mathrm{in}}(\pi)$.

Among all the statistics reported in (Claesson and Kitaev, 2008/09, Section 2), the only ones that are preserved by $P$ are the ones that depend only on the shape of in-order trees.

\section{Proof of Conjecture 1}

\subsection{Preparation}

In addition to the results of Section 2 the principal ingredients in the proof to follow are a pair of observations concerning $P$ and operators $\mathbf{A}$ which are compositions of $\mathbf{S}$ and $\mathbf{R}$.

Observation 9 Let $\tau$ be any permutation, and $\mathbf{A}$ be any composition of the operators $\mathbf{S}$ and $\mathbf{R}$. Suppose that $x, y \in[n]$ and that in $\tau$ there are no values larger than $\max (x, y)$ occurring between $x$ and $y$. Then the same holds in $\mathbf{A}(\tau)$.

Proof: It suffices to prove the result for $\mathbf{S}$ and $\mathbf{R}$ individually. For $\mathbf{R}$ it is trivial and for $\mathbf{S}$ it is not hard to prove that it follows by induction from the recursive description: $\mathbf{S}(\alpha n \beta)=\mathbf{S}(\alpha) \mathbf{S}(\beta) n$.

For the second observation we introduce a notational convention that we shall continue to use throughout. Let $\pi \in \operatorname{Av}(231)$ be given. We think of the sequence $P(\pi)$ as describing a relabeling of the values that occur in $\pi$ according to a certain permutation $\lambda_{\pi}$, specifically $P(\pi)=\lambda_{\pi} \circ \pi$. 
Observation 10 Let $\pi \in \operatorname{Av}(231)$ be given and suppose that $x, y \in[n], x<y$, and in $\pi$ there are no values larger than $\max (x, y)$ occurring between $x$ and $y$. Then $\lambda_{\pi}(x)<\lambda_{\pi}(y)$.

Proof: The proof shall not be detailed here. Observation 10 simply says that $\lambda_{\pi}$ preserves the ordering among elements of $\pi$ which do not contain a larger element between them. This follows from the construction of $P$ since the only way that one element can be moved above another one is to (at some point in the recursion) have a larger element in between.

\subsection{The main argument}

In this section we prove the main result. Recall that $\mathbf{A}$ is an operator formed by some composition of $\mathbf{S}$ and $\mathbf{R}$. For any such operator, we shall write $\pi \in \mathbf{A}$ to denote that $\pi$ is in the image of $\mathbf{A}$.

As above we consider $\lambda_{\pi}$ as a relabeling of the elements of $[n]$. We extend its effect to permutations, trees etc. that carry labels from $[n]$ : applying $\lambda_{\pi}$ to such an object will simply mean to apply $\lambda_{\pi}$ to each of its labels.

Definition 11 We define a function $\Phi_{\mathbf{A}}$ from the set of permutations sorted by $\mathbf{S} \circ \mathbf{A}$ to the set of all permutations as follows. For $\theta$ a permutation sorted by $\mathbf{S} \circ \mathbf{A}$, since $\mathbf{A}(\theta) \in \operatorname{Av}(231)$, we have $\lambda_{\mathbf{A}(\theta)}$ defined by $P(\mathbf{A}(\theta))=\lambda_{\mathbf{A}(\theta)} \circ \mathbf{A}(\theta)$ and we then set $\Phi_{\mathbf{A}}(\theta)=\lambda_{\mathbf{A}(\theta)} \circ \theta$.

In other words $\Phi_{\mathbf{A}}$ relabels a permutation $\theta$ sorted by $\mathbf{S} \circ \mathbf{A}$ in the same way that $\mathbf{A}(\theta)$ is relabeled to produce $P\left(\mathbf{A}(\theta)\right.$ ). We will prove (see Corollary 15 that $\Phi_{\mathbf{A}}$ is a bijection from the set of permutations sorted by $\mathbf{S} \circ \mathbf{A}$ to the set of those sorted by $\mathbf{S} \circ \mathbf{R} \circ \mathbf{A}$. The key to this argument of course is to establish that $\mathbf{A}\left(\Phi_{\mathbf{A}}(\theta)\right)=P(\mathbf{A}(\theta))$.

We are concerned with operators $\mathbf{A}$ which are compositions of $\mathbf{S}$ and $\mathbf{R}$. We say that such an operator respects $P$ if it has the following property:

For each $\pi \in \operatorname{Av}(231) \cap \mathbf{A}$,

- For each $\theta$ such that $\mathbf{A}(\theta)=\pi$, we have $\mathbf{A}\left(\Phi_{\mathbf{A}}(\theta)\right)=P(\pi)=\lambda_{\pi} \circ \pi$ and $\mathrm{T}_{\mathrm{in}}\left(\Phi_{\mathbf{A}}(\theta)\right)=\lambda_{\pi}\left(\mathrm{T}_{\mathrm{in}}(\theta)\right)$, and

- the correspondence $\Phi_{\mathbf{A}}: \theta \mapsto \Phi_{\mathbf{A}}(\theta)$ is a bijection between $\mathbf{A}^{-1}(\pi)$ and $\mathbf{A}^{-1}(P(\pi))$.

In the above, notice that because $\mathbf{A}(\theta)=\pi$ we actually have $\Phi_{\mathbf{A}}(\theta)=\lambda_{\pi} \circ \theta$.

Proposition 12 If $\mathbf{A}$ respects $P$ then so does $\mathbf{A} \circ \mathbf{R}$.

Proof: We shall only give the main arguments of the proof.

Let $\pi \in \operatorname{Av}(231) \cap(\mathbf{A} \circ \mathbf{R})$ and $\theta$ be such that $(\mathbf{A} \circ \mathbf{R})(\theta)=\pi$. Let $\tau=\mathbf{R}(\theta)$. Then $\mathbf{A}(\tau)=\pi$ and since $\mathbf{A}$ respects $P, \mathbf{A}\left(\Phi_{\mathbf{A}}(\tau)\right)=P(\pi)$ and $\mathrm{T}_{\mathrm{in}}\left(\Phi_{\mathbf{A}}(\tau)\right)=\lambda_{\pi}\left(\mathrm{T}_{\mathrm{in}}(\tau)\right)$.

Because $\mathbf{R}$ is an involution on permutations that acts only on positions whereas $\lambda_{\pi}$ acts on values only, it can be proved that $\mathbf{R}\left(\Phi_{\mathbf{A} \circ \mathbf{R}}(\theta)\right)=\Phi_{\mathbf{A}}(\tau)$. It follows that $(\mathbf{A} \circ \mathbf{R})\left(\Phi_{\mathbf{A} \circ \mathbf{R}}(\theta)\right)=\mathbf{A}\left(\Phi_{\mathbf{A}}(\tau)\right)=P(\pi)$. Moreover, applying $\mathbf{R}$ to a permutation is equivalent to recursively exchanging left and right subtrees in its in-order tree. This is how we deduce $\mathrm{T}_{\mathrm{in}}\left(\Phi_{\mathbf{A} \circ \mathbf{R}}(\theta)\right)=\lambda_{\pi}\left(\mathrm{T}_{\mathrm{in}}(\theta)\right)$ from $\mathrm{T}_{\mathrm{in}}\left(\Phi_{\mathbf{A}}(\tau)\right)=\lambda_{\pi}\left(\mathrm{T}_{\mathrm{in}}(\tau)\right)$. Finally, the correspondence $\Phi_{\mathbf{A} \circ \mathbf{R}}$ is the composition of three bijections: $\mathbf{R}, \Phi_{\mathbf{A}}$ and $\mathbf{R}^{-1}=\mathbf{R}$, and so is also a bijection.

Proposition 13 If $\mathbf{A}$ respects $P$ then so does $\mathbf{A} \circ \mathbf{S}$. 
Proof: For brevity, we only sketch the proof and omit the details.

Let $\pi \in \operatorname{Av}(231) \cap(\mathbf{A} \circ \mathbf{S})$ and $\theta$ be such that $(\mathbf{A} \circ \mathbf{S})(\theta)=\pi$. Let $\tau=\mathbf{S}(\theta)$. Then $\mathbf{A}(\tau)=\pi$ and since $\mathbf{A}$ respects $P, \mathbf{A}\left(\Phi_{\mathbf{A}}(\tau)\right)=P(\pi)$ and $\mathrm{T}_{\mathrm{in}}\left(\Phi_{\mathbf{A}}(\tau)\right)=\lambda_{\pi}\left(\mathrm{T}_{\mathrm{in}}(\tau)\right)$.

We first define $\tau^{\prime}=\lambda_{\pi} \circ \tau=\Phi_{\mathbf{A}}(\tau)$ and show that $\tau^{\prime} \in \mathbf{S}$. From Bousquet-Mélou (2000), we know that it is enough to prove that $\tau^{\prime}$ is the post-order reading of some decreasing binary tree. Denoting $T$ the unique canonical tree such that $\operatorname{Post}(T)=\tau$, and defining $T^{\prime}=\lambda_{\pi}(T)$, we remark that $\operatorname{Post}\left(T^{\prime}\right)=\tau^{\prime}$, hence $\tau^{\prime} \in \mathbf{S}$. Moreover, it can be proved that the tree $T^{\prime}$ is canonical, so that $T^{\prime}$ is the unique canonical tree such that $\operatorname{Post}\left(T^{\prime}\right)=\tau^{\prime}$.

Defining furthermore $\theta^{\prime}=\lambda_{\pi} \circ \theta$, we next prove that $\mathrm{T}_{\text {in }}\left(\theta^{\prime}\right)=\mathrm{T}_{\text {in }}(\theta)^{\prime}$ (i.e. the result of applying $\lambda_{\pi}$ to the labels of $\mathrm{T}_{\text {in }}(\theta)$ ) and $\mathbf{S}\left(\theta^{\prime}\right)=\tau^{\prime}$. From Bousquet-Mélou (2000) again, because $\mathbf{S}(\theta)=\tau$, we know that $\mathrm{T}_{\mathrm{in}}(\theta)$ has been obtained from $T$ by a series of moves of the following form:

Take a node $z$ with no left child, and one of its descendants $y$ on the leftmost branch of its right subtree. Remove the subtree rooted at $y$ and make it the left subtree of $z$.

Applying the same sequence of operations to $T^{\prime}$, that is, creating a tree with the same underlying structure as $T_{\text {in }}(\theta)$, but with the labels arising from $T^{\prime}$, we obtain a decreasing tree (because the operations cannot create an increasing pair) whose in-order reading is $\theta^{\prime}$, and whose post-order reading is $\tau^{\prime}$, and hence $\mathrm{T}_{\mathrm{in}}\left(\theta^{\prime}\right)=\mathrm{T}_{\mathrm{in}}(\theta)^{\prime}$ and $\mathbf{S}\left(\theta^{\prime}\right)=\tau^{\prime}$.

This implies that

- $\mathrm{T}_{\mathrm{in}}\left(\Phi_{\mathbf{A} \circ \mathbf{S}}(\theta)\right)=\mathrm{T}_{\mathrm{in}}\left(\lambda_{\pi} \circ \theta\right)=\lambda_{\pi}\left(\mathrm{T}_{\mathrm{in}}(\theta)\right)$;

- $\mathbf{A} \circ \mathbf{S}\left(\Phi_{\mathbf{A} \circ \mathbf{S}}(\theta)\right)=\mathbf{A} \circ \mathbf{S}\left(\theta^{\prime}\right)=\mathbf{A}\left(\mathbf{S}\left(\theta^{\prime}\right)\right)=\mathbf{A}\left(\tau^{\prime}\right)=\mathbf{A}\left(\Phi_{\mathbf{A}}(\tau)\right)=P(\pi)$.

The correspondence $\theta \mapsto \theta^{\prime}$ is a bijective map between $\mathbf{S}^{-1}(\tau)$ and $\mathbf{S}^{-1}\left(\tau^{\prime}\right)$ (a consequence of Proposition 2.7 of Bousquet-Mélou (2000)), and the correspondence $\Phi_{\mathbf{A} \circ \mathbf{S}}$ between $(\mathbf{A} \circ \mathbf{S})^{-1}(\pi)$ and $(\mathbf{A} \circ \mathbf{S})^{-1}(P(\pi))$ is just the union of all these correspondences on the disjoint sets $\mathbf{S}^{-1}(\tau)$ for $\tau \in \mathbf{A}^{-1}(\pi)$ and to the disjoint sets $\mathbf{S}^{-1}\left(\tau^{\prime}\right)$ for $\tau^{\prime} \in \mathbf{A}^{-1}(P(\pi))$. So it is a bijection, and $\mathbf{A} \circ \mathbf{S}$ respects $P$.

Combining the two preceding propositions with the fact that from Observation 7 the identity operator respects $P$ we obtain our main theorem:

Theorem 14 Every operator that is formed by composition from $\{\mathbf{S}, \mathbf{R}\}$ respects $P$.

Corollary 15 For any composition $\mathbf{A}$ of operators from $\{\mathbf{S}, \mathbf{R}\}, \Phi_{\mathbf{A}}$ is a bijection between the set of permutations sorted by $\mathbf{S} \circ \mathbf{A}$ and those sorted by $\mathbf{S} \circ \mathbf{R} \circ \mathbf{A}$.

Corollary 15 proves the first part of Conjecture 1 . namely that the number of permutations sorted by $\mathbf{S} \circ \mathbf{A}$ and by $\mathbf{S} \circ \mathbf{R} \circ \mathbf{A}$ is the same.

We now study the properties of bijections $\Phi_{\mathbf{A}}$ in somewhat greater detail. This will prove the second part of Conjecture 1 that deals with permutation statistics equidistributed over the set of permutations sorted by $\mathbf{S} \circ \mathbf{A}$ and the set of those sorted by $\mathbf{S} \circ \mathbf{R} \circ \mathbf{A}$.

\subsection{Statistics preserved by the bijections $\Phi_{\mathbf{A}}$}

In this section, $\mathbf{A}$ denotes any composition of operators from $\{\mathbf{S}, \mathbf{R}\}$.

Theorem 16 The shape of the in-order tree is preserved by $\Phi_{\mathbf{A}}$. 
Proof: For $\theta$ a permutation sorted by $\mathbf{S} \circ \mathbf{A}$, writing $\pi=\mathbf{A}(\theta) \in \operatorname{Av}(231)$ we have $\Phi_{\mathbf{A}}(\theta)=\lambda_{\pi} \circ \theta$. From Theorem 14. A respects $P$, so that $\mathrm{T}_{\mathrm{in}}\left(\lambda_{\pi} \circ \theta\right)$ and $\mathrm{T}_{\mathrm{in}}(\theta)$ have the same shape.

Because the shape of the in-order tree determines many permutation statistics, we have:

Corollary $17 \Phi_{\mathbf{A}}$ preserves the following statistics: the number and positions of the right-to-left maxima, the number and positions of the left-to-right maxima and the up-down word (and hence also the many classical permutation statistics determined by the up-down word).

Zeilberger (1992) introduced a statistic to aid in the enumeration of the permutations sorted by $\mathbf{S} \circ \mathbf{S}$. Unsurprisingly, this statistic and one of its close relatives is also preserved by $\Phi_{\mathbf{A}}$ :

Theorem 18 If $\mathbf{A}=\mathbf{A}_{0} \circ \mathbf{S}$ for some arbitrary composition $\mathbf{A}_{0}$ of operators from $\{\mathbf{S}, \mathbf{R}\}$, then $\Phi_{\mathbf{A}}$ preserves the Zeilberger statistic, defined as: $\operatorname{zeil}(\theta)=\max \{k \mid n(n-1) \cdots(n-k+1)$ is a subword of $\theta\}$. In addition, if there is at least one operator $\mathbf{S} \circ \mathbf{R}$ in the composition that defines $\mathbf{A}_{0}$, then $\Phi_{\mathbf{A}}$ also preserves the reverse of the above statistics: $\operatorname{Rzeil}(\theta)=\max \{k \mid(n-k+1) \cdots(n-1) n$ is a subword of $\theta\}$.

Proof: We only provide a sketch of the proof.

Consider $\theta$ a permutation sorted by $\mathbf{S} \circ \mathbf{A}$, and set $\pi=\mathbf{A}(\theta)$. Then $\Phi_{\mathbf{A}}(\theta)=\lambda_{\pi} \circ \theta$, and we may interpret this identity as $\Phi_{\mathbf{A}}(\theta)$ being obtained relabeling the elements of $\theta$ according to $\lambda_{\pi}$. As before, we extend the effect of relabeling by $\lambda_{\pi}$ to any object that carries labels from $[n]$.

For the first statement, let $c \leq n$ be the smallest value of $[n]$ such that all $d \geq c$ are unaffected by the relabeling $\lambda_{\pi}$. Because $\mathrm{T}_{\mathrm{in}}\left(\lambda_{\pi} \circ \theta\right)=\lambda_{\pi}\left(\mathrm{T}_{\mathrm{in}}(\theta)\right)$, it is not hard to see that it is enough to prove that $c \leq n-k$, where $k=\operatorname{zeil}(\theta)$. This is proved by contradiction, using the fact that $\mathbf{S}(\theta)$ is the post-order reading of $T_{\text {in }}(\theta)$, together with Observations 9 and 10 .

For the second statement, we may write $\mathbf{A}=\mathbf{B}_{0} \circ \mathbf{S} \circ \mathbf{R} \circ \mathbf{S}^{k}$, with $k \geq 1$. Then, we apply the first statement to $\mathbf{B}_{0} \circ \mathbf{S}$, and we notice that $\mathbf{R}$ maps the zeil statistics to Rzeil. To conclude the proof, the most important fact is that applying operator $\mathbf{S}$ may only increase the value of the Rzeil statistics.

\section{More properties of the bijection $P$}

\subsection{Bijection $P$ and Wilf-equivalences}

Two permutation classes are said to be Wilf-equivalent if they contain the same number of permutations of length $n$ for every $n$. One common form of Wilf-equivalence arises from symmetries of the avoidance relationship. For example, the reverse symmetry $\mathbf{R}$ provides a bijection between $\operatorname{Av}(231)$ and $\operatorname{Av}(132)$, proving that these classes are Wilf-equivalent. More generally, for any symmetry $\mathbf{Z}$ obtained composing reverse, complement and inverse, $\operatorname{Av}\left(\pi, \pi^{\prime}, \cdots, \pi^{\prime \prime}\right)$ and $\operatorname{Av}\left(\mathbf{Z}(\pi), \mathbf{Z}\left(\pi^{\prime}\right), \cdots, \mathbf{Z}\left(\pi^{\prime \prime}\right)\right)$ are Wilf-equivalent classes, and we say that they are trivially Wilf-equivalent. However, more interesting Wilf-equivalences are also somewhat common, and in this section we show how the bijection $P$ from Section 3 furnishes a supply of such Wilf-equivalences.

We say that a permutation $\pi \in \operatorname{Av}(231)$ respects $P$ when $P$ restricted to $\operatorname{Av}(231, \pi)$ is a bijection with $\operatorname{Av}(132, P(\pi))$. We define two families of permutations $\left(\lambda_{n}\right)$ and $\left(\rho_{n}\right)$ recursively by $\lambda_{1}=\rho_{1}=1$ and for all $n \geq 1, \lambda_{n+1}=1 \ominus \rho_{n}$ and $\rho_{n+1}=\lambda_{n} \oplus 1$ (see Figure 4). We also take the convention that $\lambda_{0}$ and $\rho_{0}$ denote the empty permutation. Notice that for any $n, \lambda_{n}$ and $\rho_{n}$ are fixed by $P$, since they avoid both 231 and 132. Notice also that for any $n, \lambda_{n}$ is $\oplus$-indecomposable and $\rho_{n}$ is $\ominus$-indecomposable. 


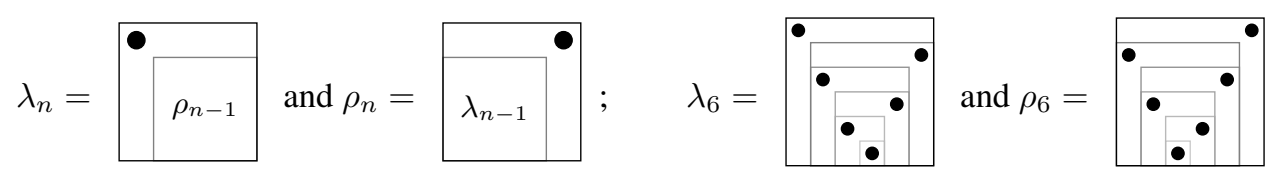

Fig. 4: Diagrams of $\lambda_{n}$ and $\rho_{n}$, for general $n$ and for $n=6$.

Lemma 19 For every $n \geq 0$, and every $0 \leq k \leq n$, the permutation $\lambda_{k} \oplus \lambda_{n-k}$ respects $P$.

Proof: The proof of this result is based on an induction on $n$, and simply requires a careful analysis of the way in which a pattern such as $\lambda_{k} \oplus \lambda_{n-k}$ can occur in a 231-avoiding permutation, and dually how $P\left(\lambda_{k} \oplus \lambda_{n-k}\right)$ can occur in a 132 -avoiding permutation.

Since $\lambda_{n}$ and $\rho_{n}$ are fixed by $P$, and because $\lambda_{k} \oplus \lambda_{n-k}=\lambda_{k} \oplus\left(1 \ominus \rho_{n-k-1}\right)$, a consequence of Lemma19 is:

Theorem 20 For every $n \geq 0$, and every $0 \leq k \leq n-1$, the permutation classes $\operatorname{Av}\left(231, \lambda_{k} \oplus(1 \ominus\right.$ $\left.\left.\rho_{n-k-1}\right)\right)$ and $\operatorname{Av}\left(132,\left(\lambda_{k} \oplus 1\right) \ominus \rho_{n-k-1}\right)$ are Wilf-equivalent. Moreover, $P$ provides a bijection from one to the other, that preserves the shape of the in-order trees.

Even though there are more classes $\operatorname{Av}(231, \pi)$ and $\operatorname{Av}(132, P(\pi))$ that are Wilf-equivalent, we are able to show that except when $\pi$ of the form of Lemma 19, $P$ will not provide a bijection between $\operatorname{Av}(231, \pi)$ and $\operatorname{Av}(132, P(\pi))$. This is obtained proving the converse of Lemma 19, i.e. proving that all permutations that respect $P$ are of the form $\lambda_{k} \oplus \lambda_{n-k}$. The proof is omitted for brevity.

Theorem 21 The permutations that respect $P$ are exactly those of the form $\lambda_{k} \oplus \lambda_{n-k}=\lambda_{k} \oplus(1 \ominus$ $\left.\rho_{n-k-1}\right)$, for $n \geq 0$ and $0 \leq k \leq n-1$.

Table 1 shows all patterns that respect $P$ of length 3 to 8 . To each such pattern corresponds a Wilfequivalence between $\operatorname{Av}(231, \pi)$ and $\operatorname{Av}(132, P(\pi))$. They are non trivial, except for three of them that correspond to the reverse symmetry - those are indicated in italics. Due to symmetries, some Wilfequivalences may however correspond to several rows in Table 1. For instance, $\pi=42135$ and $\pi^{\prime}=$ 53124 yield the same Wilf-equivalence up to a reverse symmetry.

For $\pi$ of length 3 or 4 , the Wilf-equivalences obtained from Table 1 may be compared to those reported in Wikipedia (2013). Among the Wilf-equivalences reported therein that we may hope to recover (i.e. when one of the excluded pattern is 231 or one of its symmetries), we find three of them, while five are left aside. These three are:

- because $P(312)=312, \operatorname{Av}(231,312)$ is Wilf-equivalent to $\operatorname{Av}(132,312)$;

- because $P(3124)=3124, \operatorname{Av}(231,3124)$ is Wilf-equivalent to $\operatorname{Av}(132,3124)$ which is up to reverse symmetry the same as $\operatorname{Av}(132,4213)$ being Wilf-equivalent to $\operatorname{Av}(132,3124)$;

- because $P(1423)=3412, \operatorname{Av}(231,1423)$ is Wilf-equivalent to $\operatorname{Av}(132,3412)$ which is up to inverse-complement symmetry the same as $\operatorname{Av}(132,4213)$ being Wilf-equivalent to $\operatorname{Av}(132,3412)$.

Computer experiments have shown that there are (conjecturally) other Wilf-equivalences between classes $\operatorname{Av}(231, \pi)$ and $\operatorname{Av}(132, P(\pi))$, where $\pi$ does not respect $P$. These are shown in Table 2. 


\begin{tabular}{|l|l|}
\hline$\pi$ & $P(\pi)$ \\
\hline \hline 213 & 213 \\
\hline 132 & 231 \\
\hline 312 & 312 \\
\hline
\end{tabular}

\begin{tabular}{|l|l|}
\hline$\pi$ & $P(\pi)$ \\
\hline \hline 2143 & 3241 \\
\hline 1423 & 3412 \\
\hline 4213 & 4213 \\
\hline 3124 & 3124 \\
\hline
\end{tabular}

\begin{tabular}{|l|l|}
\hline$\pi$ & $P(\pi)$ \\
\hline \hline 42135 & 42135 \\
\hline 21534 & 43512 \\
\hline 53124 & 53124 \\
\hline 31254 & 42351 \\
\hline 15324 & 45213 \\
\hline
\end{tabular}

\begin{tabular}{|l|l|}
\hline$\pi$ & $P(\pi)$ \\
\hline \hline 216435 & 546213 \\
\hline 531246 & 531246 \\
\hline 312645 & 534612 \\
\hline 642135 & 642135 \\
\hline 421365 & 532461 \\
\hline 164235 & 563124 \\
\hline
\end{tabular}

\begin{tabular}{|l|l|}
\hline$\pi$ & $P(\pi)$ \\
\hline \hline 6421357 & 6421357 \\
\hline 3127546 & 6457213 \\
\hline 7531246 & 7531246 \\
\hline 4213756 & 6435712 \\
\hline 1753246 & 6742135 \\
\hline 5312476 & 6423571 \\
\hline 2175346 & 6573124 \\
\hline
\end{tabular}

\begin{tabular}{|l|l|}
\hline$\pi$ & $P(\pi)$ \\
\hline \hline 31286457 & 75683124 \\
\hline 75312468 & 75312468 \\
\hline 64213587 & 75324681 \\
\hline 53124867 & 75346812 \\
\hline 86421357 & 86421357 \\
\hline 21864357 & 76842135 \\
\hline 42138657 & 75468213 \\
\hline 18642357 & 78531246 \\
\hline
\end{tabular}

Tab. 1: Pairs of patterns $(\pi, P(\pi))$ such that $\pi$ respects $P$, i.e. such that $P$ provides a bijection between $\operatorname{Av}(231, \pi)$ and $\operatorname{Av}(132, P(\pi))$. In particular, these classes are Wilf-equivalent.

\begin{tabular}{|l|l|}
\hline$\pi$ & $P(\pi)$ \\
\hline \hline 2137465 & 5467231 \\
\hline 1327645 & 5647312 \\
\hline
\end{tabular}

\begin{tabular}{|l|l|}
\hline$\pi$ & $P(\pi)$ \\
\hline \hline 63125478 & 64235178 \\
\hline 87153246 & 87452136 \\
\hline 65312478 & 65312478 \\
\hline 87421356 & 87421356 \\
\hline
\end{tabular}

Tab. 2: The other patterns $\pi$ up to length 8 such that $\operatorname{Av}(231, \pi)$ and $\operatorname{Av}(132, P(\pi))$ are (conjecturally) Wilfequivalent.

\subsection{Enumeration of $\operatorname{Av}(231, \pi)$, for $\pi$ respecting $P$}

Theorem 20 shows that for any $n$, there are $n$ permutations $\pi \in \operatorname{Av}_{n}(231)$ such that the two classes $\operatorname{Av}(231, \pi)$ and $\operatorname{Av}(132, P(\pi))$ are Wilf-equivalent. We can actually prove that these $2 n$ permutation classes we obtain (as exemplified in Table 1 are all Wilf-equivalent. Notice that for both $n=7$ and 8 , all classes $\operatorname{Av}(231, \pi)$ with $\pi$ of length $n$ in Table 2 are not in the same Wilf-equivalence class.

The above Wilf-equivalence result follows immediately from Theorem 24 below. We first define a family of generating function $F_{n}(t)$ recursively as follows: $F_{1}(t)=1$, and for $n \geq 1$

$$
F_{n+1}(t)=\frac{1}{1-t F_{n}(t)} \text { for } n \geq 1
$$

This family satisfies a property that we shall use in the proof of Theorem 24

Lemma 22 Define $g(x, y)=\frac{1-t x y}{1-t x-t y}$. For any $n \geq 3$, and any $j, k \geq 1$ such that $j+k=n-1$, $F_{n}=g\left(F_{j}, F_{k}\right)$. 
Proof: Fix some $n \geq 3$. Let us remark that $g\left(\frac{1}{1-t x}, \frac{y-1}{t y}\right)=g(x, y)$.

Consequently, for any $j>1$ and $k=n-j-1$, we have $g\left(F_{j}, F_{k}\right)=g\left(F_{j-1}, F_{k+1}\right)$. So it is enough to prove that $g\left(F_{1}, F_{n-2}\right)=F_{n}$. It is easily derived from the definition of the family $\left(F_{n}\right)$. that:

Based on the decompositions $\lambda_{n}=1 \ominus \rho_{n-1}$ and $\rho_{n}=\lambda_{n-1} \oplus 1$ it is relatively easy to prove inductively

Lemma 23 The generating functions of $\operatorname{Av}\left(231, \lambda_{n}\right)$ and $\operatorname{Av}\left(231, \rho_{n}\right)$ respectively are both equal to $F_{n}$.

Finally we can also establish using the preceding two results:

Theorem 24 Let $\pi \in \operatorname{Av}_{n}(231)$ be a permutation that respects $P$. The generating function of $\operatorname{Av}(231, \pi)$ is $F_{n}$.

Proof: This follows immediately from Lemma 23 if $\pi$ is of the form $\lambda_{n}$ or $\rho_{n}$ for any $n \geq 1$. Otherwise, by Theorem 21, we have $\pi=\lambda_{j} \oplus\left(1 \ominus \rho_{k}\right)$ for some $j \geq 1$ and $k \geq 1$. Let $\mathcal{C}=\operatorname{Av}(231, \pi)$ and let $C$ be the corresponding generating function. When decomposing permutations of $\mathcal{C}$ as $\alpha \oplus(1 \ominus \beta)$, the subsequent constraints on $\alpha$ and $\beta$, together with Lemma 23 , allow us to write that

$$
C=1+t F_{j} C+t\left(C-F_{j}\right) F_{k}, \text { i.e. } C=\frac{1-t F_{j} F_{k}}{1-t F_{j}-t F_{k}} .
$$

Lemma 22 then ensures that $C=F_{n}$.

\section{Conclusions}

Many other permutation classes have recursive descriptions similar to those of $\operatorname{Av}(231)$ and $\operatorname{Av}(132)$. In such cases it may well be possible to define analogous bijections to $P$ which could lead to a unified framework for understanding Wilf-equivalences between their subclasses. Indeed, even for these two classes it is possible to combine the bijections $P$ and $\mathbf{R}$ into various hybrid forms, and some of these may be useful in characterising the additional Wilf-equivalences that seem to exist in this context.

Of course our results provide some bijections between collections of permutations sorted by some combinations of $\mathbf{S}$ and $\mathbf{R}$. However, they do not provide enumerations of these collections - this seems to remain a difficult problem in general (and even more so if symmetries other than $\mathbf{R}$ are included) as suggested by the relative difficulty of enumerating the permutations sorted by $\mathbf{S} \circ \mathbf{S}$ compared to those sorted by $\mathbf{S}$. Another point is to determine whether or not the bijection here between specifically the permutations sorted by $\mathbf{S} \circ \mathbf{S}$ and those sorted by $\mathbf{S} \circ \mathbf{R} \circ \mathbf{S}$ is the same as the one described implicitly in Bouvel and Guibert (2012).

There are other relatively natural sorting operators. For instance bubble sort can be defined by $\mathrm{B}(\alpha n \beta)=$ $\mathrm{B}(\alpha) \beta n$. Albert et al. (2011) considered the inverse images of permutation classes under $\mathrm{B}$ and some investigations of composites of $B$ and related operators have been reported by Ferrari (2012). Combining such operators with $\mathbf{S}$ (and other possibilities) offers further scope for the discovery (or explanation) of Wilf-equivalences among permutation classes. 


\section{Acknowledgements}

Many thanks to Olivier Guibert whose experimental investigations were critical in framing the questions addressed in this paper. Much of the work in this paper was supported by the software suite PermLab Albert (2012), and extensions of it. Michael Albert would like to thank LaBRI for their hospitality and support in August and September of 2012 when most of this work was carried out.

\section{References}

M. Albert. Permlab: Software for permutation patterns. http://www.cs.otago.ac.nz/ PermLab/, 2012.

M. H. Albert, M. D. Atkinson, M. Bouvel, A. Claesson, and M. Dukes. On the inverse image of pattern classes under bubble sort. J. Comb., 2(2):231-243, 2011.

M. Bousquet-Mélou. Sorted and/or sortable permutations. Discrete Math., 225(1-3):25-50, 2000.

M. Bouvel and O. Guibert. Enumeration of permutations sorted with two passes through a stack and $D_{8}$ symmetries. DMTCS Proceedings, 0(01), 2012.

A. Claesson and S. Kitaev. Classification of bijections between 321- and 132-avoiding permutations. Sém. Lothar. Combin., 60:Art. B60d, 30p., 2008/09.

L. S. Ferrari. Commutation properties among bubblesort, stacksort and their duals. Proceedings of GASCom'12, Bordeaux, 2012.

D. E. Knuth. The art of computer programming. Addison-Wesley Publishing Co., Reading, Mass.London-Amsterdam, second edition, 1975. Volume 1: Fundamental algorithms, Addison-Wesley Series in Computer Science and Information Processing.

V. R. Pratt. Computing permutations with double-ended queues. Parallel stacks and parallel queues. In Fifth Annual ACM Symposium on Theory of Computing (Austin, Tex., 1973), pages 268-277. Assoc. Comput. Mach., New York, 1973.

R. Tarjan. Sorting using networks of queues and stacks. J. Assoc. Comput. Mach., 19:341-346, 1972.

J. West. Sorting twice through a stack. Theoret. Comput. Sci., 117(1-2):303-313, 1993.

Wikipedia. Enumerations of specific permutation classes. http://en.wikipedia.org/wiki/ Enumerations_of_specific_permutation_classes, accessed on April 12, 2013.

D. Zeilberger. A proof of Julian West's conjecture that the number of two-stack-sortable permutations of length $n$ is $2(3 n) ! /((n+1) !(2 n+1) !)$. Discrete Math., 102(1):85-93, 1992. 